4-1-2015

\title{
Staff Perceptions of the Barriers to Mobilizing ICU Patients
}

Simone Dafoe

Royal Adelaide Hospital

Kathy Stiller

Royal Adelaide Hospital, kathy.stiller@health.sa.gov.au

Marianne Chapman

Royal Adelaide Hospital

Follow this and additional works at: https://nsuworks.nova.edu/ijahsp

Part of the Physical Therapy Commons, and the Physiotherapy Commons

\section{Recommended Citation}

Dafoe S, Stiller K, Chapman M. Staff Perceptions of the Barriers to Mobilizing ICU Patients. The Internet Journal of Allied Health Sciences and Practice. 2015 Apr 01;13(2), Article 8.

This Manuscript is brought to you for free and open access by the College of Health Care Sciences at NSUWorks. It has been accepted for inclusion in Internet Journal of Allied Health Sciences and Practice by an authorized editor of NSUWorks. For more information, please contact nsuworks@nova.edu. 


\title{
Staff Perceptions of the Barriers to Mobilizing ICU Patients
}

\begin{abstract}
Purpose: Whilst early progressive mobilization is known to be safe and beneficial for patients in an intensive care unit (ICU), barriers still exist to its implementation. As part of a broader quality improvement project that had the overall aim of increasing the frequency of mobilization in our ICU, we conducted a survey of ICU staff to investigate their perceptions of the barriers to the early progressive mobilization of ICU patients. Method: A prospective survey of ICU staff in an Australian, tertiary care, public hospital ICU was undertaken. A total of 93 medical, nursing, and physiotherapy staff participated. A purposedesigned survey that investigated staff perceptions of the barriers to the early progressive mobilization of ICU patients was developed. The survey predominantly comprised closed statements requiring responses using a visual analogue scale. Barriers to early progressive mobilization were separated into three sections: patient-related, institutional-related, and other barriers. Results: Patient-related barriers were generally perceived as having the greatest influence on the mobilization of ICU patients, followed closely by institutional-related barriers. The factors that were perceived as most frequently preventing mobilization were hemodynamic instability, reduced level of consciousness, sedation, agitation, impending medical procedure, staff availability, and time constraints. Conclusions: ICU staff perceived that barriers to the early progressive mobilization of ICU patients were multifactorial and most frequently involved patients' medical condition and resource limitations.
\end{abstract}




\title{
TIUAHSP
}

\section{The Internet Joưnal of Allied Health Sciences and Practice}

Dedicated to allied health professional practice and education

Vol. 13 No. 2 ISSN 1540-580X

\section{Staff Perceptions of the Barriers to Mobilizing ICU Patients}

\author{
Simone Dafoe - BAppSc (Physio) ${ }^{1}$ \\ Kathy Stiller - BAppSc (Physio), PhD2 \\ Marianne Chapman - BMBS, PhD, FFARCSI, FANZCA, FCICM ${ }^{3}$
}

1. Principal Physiotherapist, Acute Care and Surgery, Physiotherapy Department, Royal Adelaide Hospital, Adelaide, South Australia.

2. Allied Health Research Coordinator, Royal Adelaide Hospital, Adelaide, South Australia.

3. Director of Research and Senior Consultant, Intensive Care Unit, Royal Adelaide Hospital, Adelaide, South Australia.

Australia

\begin{abstract}
Purpose: Whilst early progressive mobilization is known to be safe and beneficial for patients in an intensive care unit (ICU), barriers still exist to its implementation. As part of a broader quality improvement project that had the overall aim of increasing the frequency of mobilization in our ICU, we conducted a survey of ICU staff to investigate their perceptions of the barriers to the early progressive mobilization of ICU patients. Method: A prospective survey of ICU staff in an Australian, tertiary care, public hospital ICU was undertaken. A total of 93 medical, nursing, and physiotherapy staff participated. A purpose-designed survey that investigated staff perceptions of the barriers to the early progressive mobilization of ICU patients was developed. The survey predominantly comprised closed statements requiring responses using a visual analogue scale. Barriers to early progressive mobilization were separated into three sections: patient-related, institutional-related, and other barriers. Results: Patient-related barriers were generally perceived as having the greatest influence on the mobilization of ICU patients, followed closely by institutional-related barriers. The factors that were perceived as most frequently preventing mobilization were hemodynamic instability, reduced level of consciousness, sedation, agitation, impending medical procedure, staff availability, and time constraints. Conclusions: ICU staff perceived that barriers to the early progressive mobilization of ICU patients were multifactorial and most frequently involved patients' medical condition and resource limitations.
\end{abstract}

\section{INTRODUCTION}

There is increasing evidence demonstrating that early progressive mobilization of patients in the intensive care unit (ICU) is safe, with a low rate of adverse events, and beneficial in terms of its positive effect on patients' functional ability and its potential to reduce ICU and hospital length of stay. ${ }^{1-7}$ Despite this evidence, barriers to the mobilization of ICU patients have been identified which have the potential to decrease the uptake of research findings into clinical practice. These barriers include patient-related factors such as cardiovascular, respiratory and/or neurological instability, level of consciousness, pain/discomfort, and the site of vascular/other invasive lines.,2,-22 Institutional-related barriers include sedation practices, a conservative ICU culture where mobilization is under-valued, uncertainty regarding professional responsibility for mobilization (e.g., physiotherapists or nurses), poor interdisciplinary cooperation, insufficient staffing/resources, and fear of staff injury. 8,10-14,16-18,20-24

These barriers to the mobilization of ICU patients were predominantly identified from data reported in clinical studies or staff surveys investigating broad mobilization/rehabilitation practices in the ICU.,2,8,911,12,14-16,18-22,24 We were unable to identify any staff survey that specifically focused and explored, in detail, staff perceptions of the barriers to the mobilization of ICU patients. We believe this is an important oversight, as an understanding of ICU staff's perceptions of the barriers to mobilization may identify those barriers that are amenable to intervention, thus providing opportunities to increase the frequency of mobilization of ICU patients and, in doing so, narrow the gap between evidence and clinical practice. An increased frequency of mobilization of ICU patients should improve patients' function and potentially decrease ICU and hospital length of stay. Therefore, the aim of this 
study, which was part of a broader quality improvement project aimed at increasing the frequency of mobilization in our ICU, was to evaluate ICU staff perceptions of the barriers to mobilizing ICU patients.

\section{METHODS}

Design

A prospective survey was undertaken as part of a broader quality improvement project. The study was approved by the Royal Adelaide Hospital Research Ethics Committee and registered with the Australian New Zealand Clinical Trials Registry.

\section{Setting and Participants}

The study was conducted at the Royal Adelaide Hospital, a 650-bed, tertiary care, urban, public hospital in Australia over a two month period (December 2013 to January 2014). The ICU at the Royal Adelaide Hospital has 32 ICU and 10 flexible ICU/ high dependency unit beds. These beds cater for approximately 1,500 ICU admissions per annum, composed of a mixture of general medical, general surgical, cardiothoracic, trauma, neurosurgical, spinal injury, and burns cases. Eligible participants comprised all permanent/semi-permanent medical staff (i.e., consultants or senior registrars), nursing staff (registered or enrolled nurses), and physiotherapists working in the Royal Adelaide Hospital ICU. Potential participants were identified from staffing lists, and a paper copy of the survey was sent to them via the internal mail system.

\section{Outcome}

During the design stage, we were unable to identify any published survey that was suitable for direct use or modification. Hence we developed a purpose-designed survey to investigate staff perceptions of the barriers to the early progressive mobilization of ICU patients. Various relevant themes and issues discussed in previous clinical studies and surveys were identified by the investigators and incorporated into the purpose-designed survey (e.g., patient-related barriers such as medical stability, institutional-related barriers such as staffing/resources). $.8,810-23$ Drafts of the survey were cycled between the investigators to identify and fine-tune content and improve clarity. The final draft was given to a single senior medical, nursing, and physiotherapy ICU staff member and their comprehensive feedback sought; their feedback was incorporated to produce the final version.

The final survey (Appendix I) consisted of an introductory section that sought basic descriptive information in order to describe the study sample (e.g., profession, duration of working in the Royal Adelaide Hospital ICU). The main body of the survey comprised a series of closed statements regarding the perceived barriers to the early progressive mobilization of ICU patients, requiring responses using a $10 \mathrm{~cm}$ visual analogue scale (VAS). These barriers were separated into three sections: patient, institutional, and other barriers. Finally, there were two items inviting open comments regarding these barriers or any others not mentioned, and suggestions for most effectively increasing the frequency of mobilization in the Royal Adelaide Hospital ICU.

An internal/external mail envelope was provided for return of the survey, and to encourage honesty in responses, this was addressed to one of the investigators who is independent from the Royal Adelaide Hospital ICU (KS). Return of the completed survey was taken as an indication of a willingness to participate.

\section{Data Analysis}

Data were transcribed onto an Excel spreadsheet and imported in SPSS for descriptive analysis. As VAS data were not normally distributed, medians and $25^{\text {th }}$ and $75^{\text {th }}$ percentiles (Tukey's Hinges) have been reported. For the two items where open comments were requested, two of the investigators (SD, KS) reviewed the responses together and, in a collaborative process, identified common themes.

\section{RESULTS}

\section{Participants}

Three-hundred-and-twenty-one staff were eligible for participation and sent a survey. Ninety-three (29\%) returned a completed survey, with the rate of return varying according to professional group (Figure 1). Reflecting our staff profile, the largest professional group was nursing, followed by medical and physiotherapy (Table 1). Virtually all participants worked in clinical roles and had worked in the Royal Adelaide Hospital ICU for at least one year (Table 1). 
Figure 1. Flow of Participants through the Study

\begin{tabular}{|c|c|c|}
\hline $\begin{array}{l}\text { Medical staff } \\
n=26\end{array}$ & $\begin{array}{l}\text { Eligible for participation } \\
\begin{array}{l}\text { Nursing staff } \\
n=291\end{array}\end{array}$ & $\begin{array}{l}\text { Physiotherapy staff } \\
n=4\end{array}$ \\
\hline \multicolumn{3}{|c|}{$\downarrow$} \\
\hline $\begin{array}{l}\text { Medical staff } \\
n=10(38 \%)\end{array}$ & $\begin{array}{l}\text { Completed survey } \\
\text { Nursing staff } \\
n=79(27 \%)\end{array}$ & $\begin{array}{l}\text { Physiotherapy staff } \\
n=4(100 \%)\end{array}$ \\
\hline
\end{tabular}

Table 1. Characteristics of the 93 Participants According To Professional Group: Number (\%)*

\begin{tabular}{|c|c|c|c|}
\hline & $\begin{array}{l}\text { Medical Staff } \\
n=10\end{array}$ & $\begin{array}{l}\text { Nursing Staff } \\
n=79\end{array}$ & $\begin{array}{l}\text { Physiotherapy Staff } \\
n=4\end{array}$ \\
\hline Professional Category & $\begin{array}{l}\text { Consultant: } 6 \text { (60) } \\
\text { Registrar: } 4 \text { (40) }\end{array}$ & $\begin{array}{l}\text { Critical care nurse: } 46 \text { (58) } \\
\text { Registered nurse: } 30 \text { (38) } \\
\text { Enrolled nurse: } 3(4)\end{array}$ & $\begin{array}{l}\text { Principal: } 2 \text { (50) } \\
\text { Senior: } 1(25) \\
\text { Base grade: } 1 \text { (25) }\end{array}$ \\
\hline $\begin{array}{l}\text { Workload } \\
\qquad \text { Clinical } \\
\text { Non-clinical }\end{array}$ & $\begin{array}{l}9(90) \\
1(10)\end{array}$ & $\begin{array}{l}77(97) \\
2(3)\end{array}$ & $\begin{array}{l}4(100) \\
0(0)\end{array}$ \\
\hline $\begin{array}{l}\text { Duration of Working in } \\
\text { the RAH ICU } \\
\quad<1 \text { year } \\
1-5 \text { years } \\
>5 \text { years }\end{array}$ & $\begin{array}{l}0 \\
4(40) \\
6(60)\end{array}$ & $\begin{array}{l}1(1) \\
32(41) \\
46(58)\end{array}$ & $\begin{array}{l}2(50) \\
2(50) \\
0(0)\end{array}$ \\
\hline
\end{tabular}

${ }^{*}$ Percentages are calculated according to each professional group.

$\mathrm{RAH}=$ Royal Adelaide Hospital; ICU = intensive care unit.

\section{Main Results}

The first item within the main part of the survey asked participants to indicate on a VAS how well they thought the Royal Adelaide Hospital ICU was doing in terms of mobilizing patients who were suitable for mobilization $(0=$ poorly, not many patients who should be mobilized are being mobilized and $10=$ excellently, all patients who should be mobilized are being mobilized). A median value of 7 was found (range $=1-10,25^{\text {th }}$ percentile $=5,75^{\text {th }}$ percentile $=8$ ).

Summary VAS data regarding staff perception of patient-related barriers, institutional-related barriers and other barriers to early progressive mobilization are shown in Figures 2-4. Overall, the range of responses for most items was wide. 
Figure 2. Visual Analogue Scale Data for Patients-Related Barriers to Mobilization

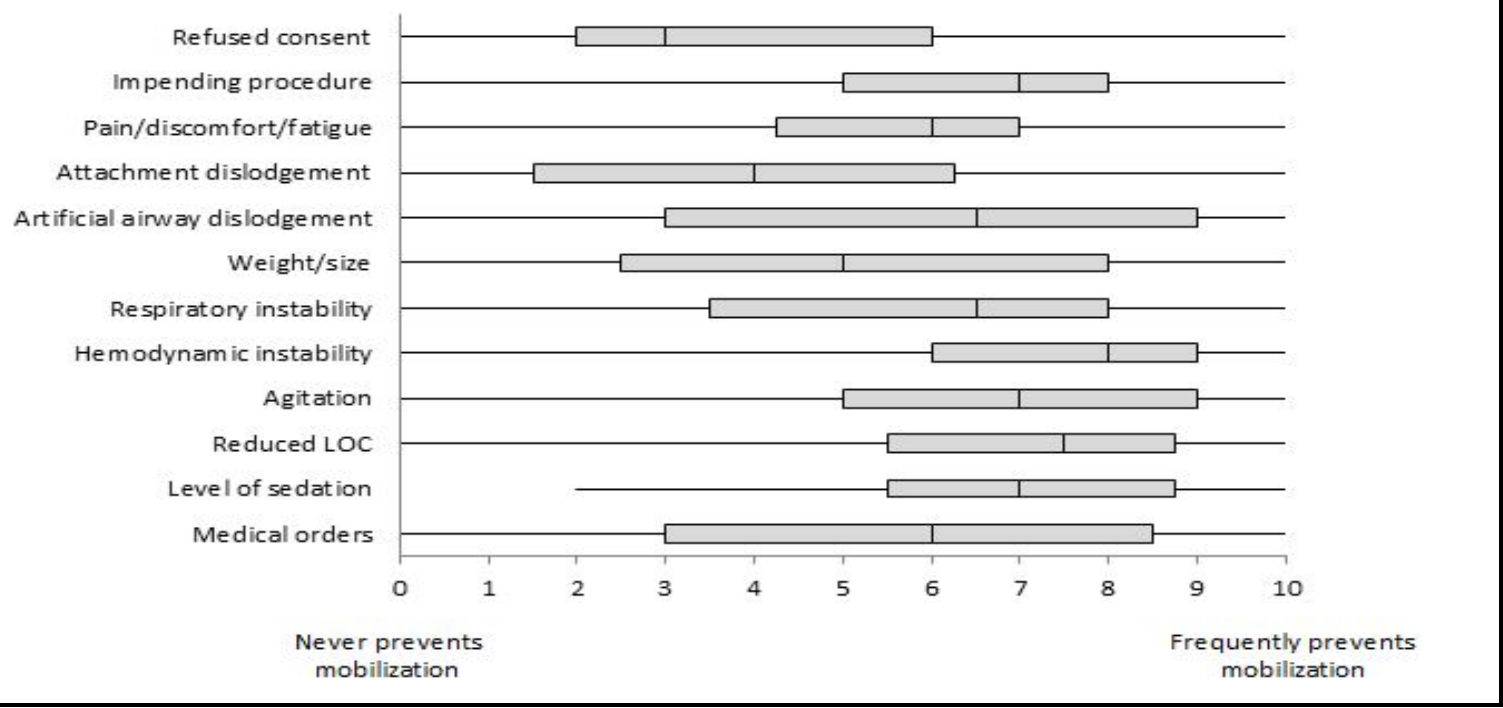

Boxes depict $25^{\text {th }}$ percentile, median and $75^{\text {th }}$ percentile. Whiskers depict minimum and maximum values.

For the patient-related barriers, the items with the highest medians (i.e., perceived as most frequently preventing mobilization) related to the patient's medical condition (e.g., hemodynamic instability, reduced level of consciousness) and impending medical procedures/investigations. Refused consent and the potential for dislodgement of vascular lines/other attachments had the lowest medians (i.e., perceived as less frequently preventing mobilization).

\section{Figure 3. Visual Analogue Scale Data for Institutional-Related Barriers to Mobilization}

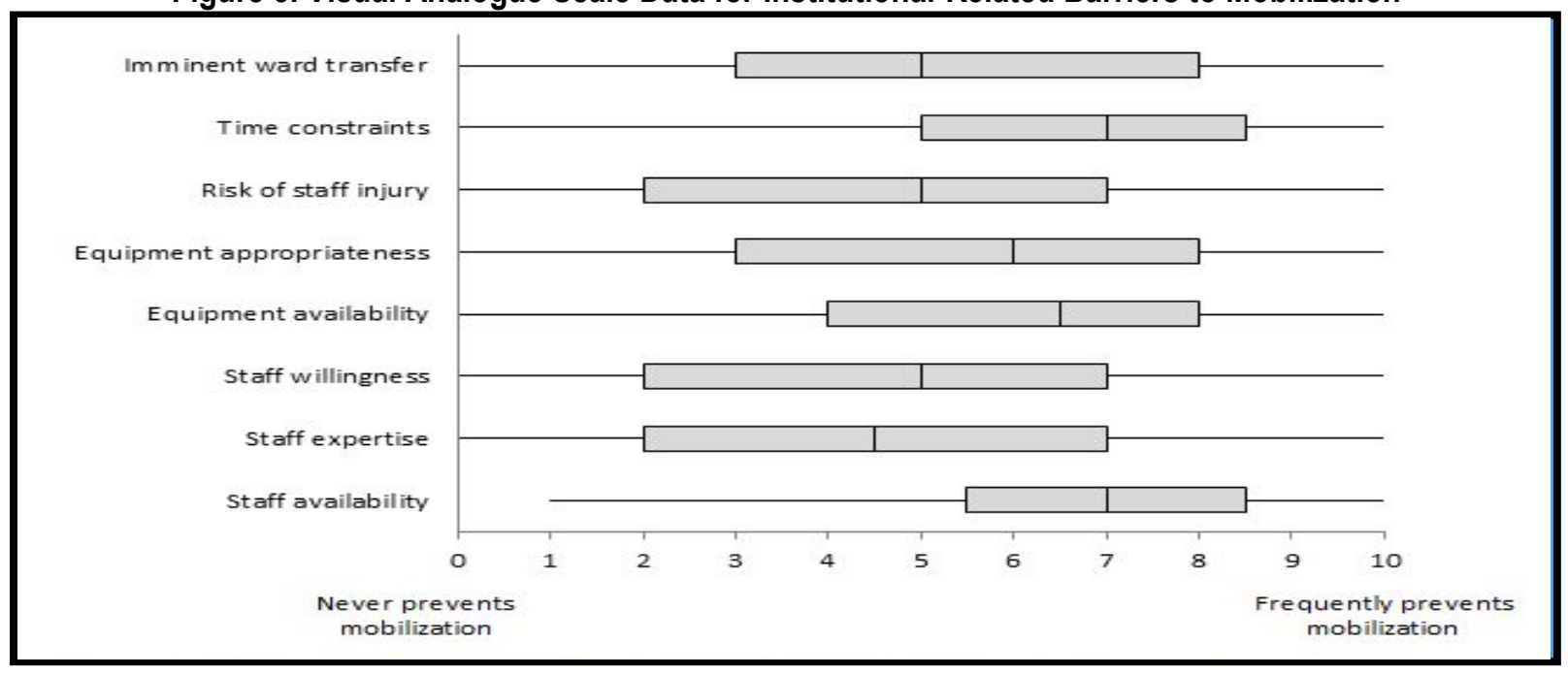

Boxes depict $25^{\text {th }}$ percentile, median and $75^{\text {th }}$ percentile. Whiskers depict minimum and maximum values.

The institutional-related barriers that were perceived by staff as most frequently preventing mobilization were time constraints and staff/equipment availability. The lowest medians, indicating items perceived as being less likely to prevent mobilization, were seen for staff expertise/willingness, risk of staff injury, and imminent ward transfer.

The medians for the other barriers to mobilization were generally lower than those seen for the patient- or institutional-related barriers. In this section, the items perceived as most frequently preventing mobilization were as follows: no clear recommendations regarding mobilization in the Royal Adelaide Hospital ICU, poor inter-disciplinary communication, and a lack of 
leadership championing the importance of mobilization. The lowest medians, indicating items perceived as being less likely to prevent mobilization, were seen for the following: that physiotherapists should have sole responsibility for all mobilization interventions, that compared to other aspects of care mobilization is unimportant, and that the Royal Adelaide Hospital ICU does not promote/value mobilization.

Figure 4. Visual Analogue Scale Data for Other Barriers to Mobilization

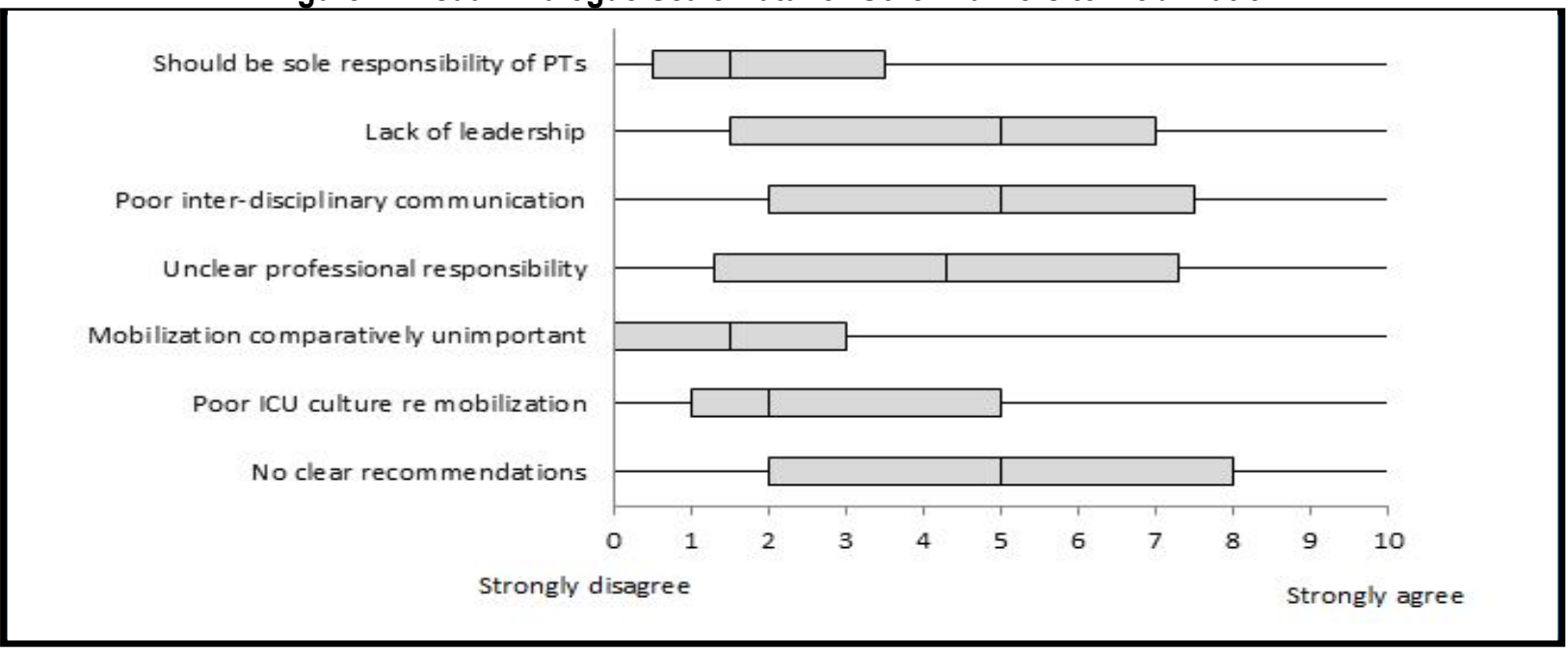

PTs = physiotherapists; ICU = intensive care unit

Boxes depict $25^{\text {th }}$ percentile, median and $75^{\text {th }}$ percentile. Whiskers depict minimum and maximum values.

In order to compare their relative importance, means were calculated for the patient-related, institutional-related and other barriers. As shown in Figure 5, patient-related barriers had the highest mean, followed by institutional-related barriers and other barriers.

Figure 5. Summary of Visual Analogue Scale Data for the Barriers to Mobilization

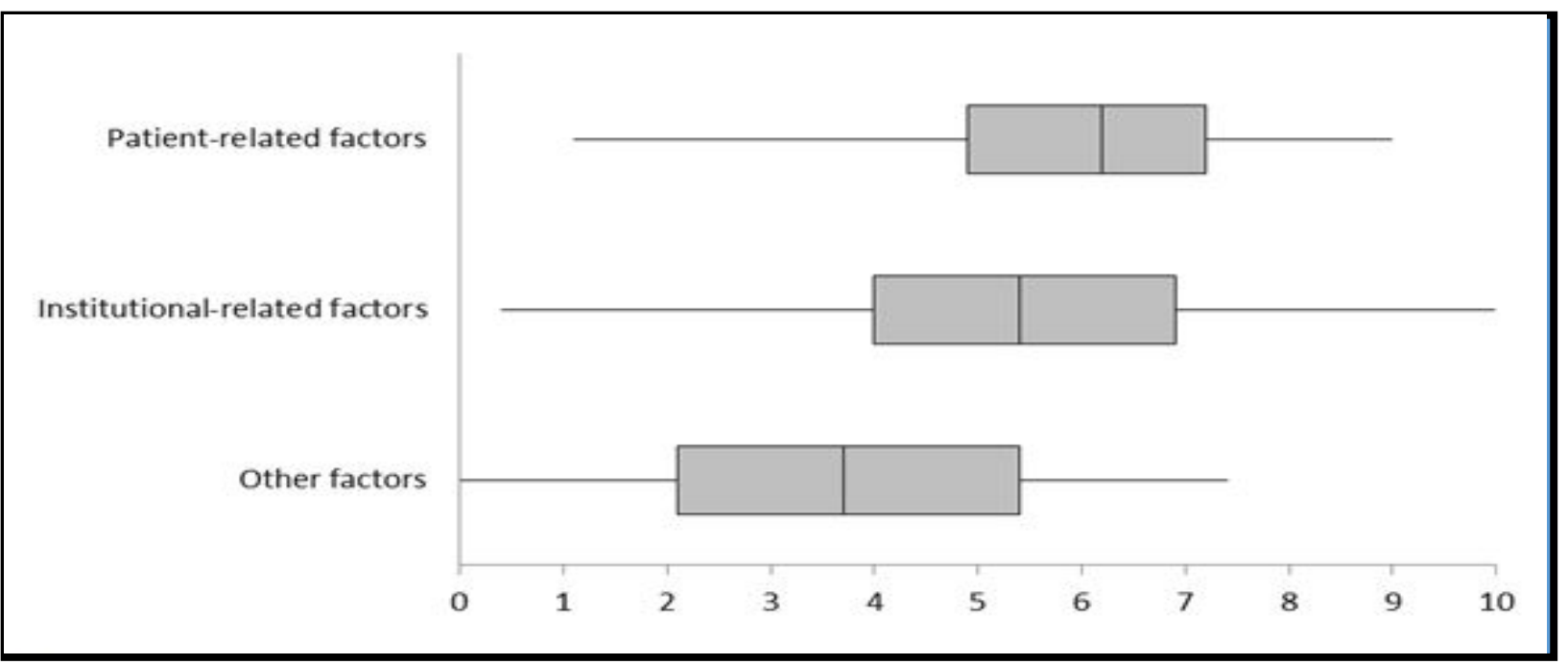

Boxes depict $25^{\text {th }}$ percentile, median and $75^{\text {th }}$ percentile. Whiskers depict minimum and maximum values

\section{Open, Free-Text Items}

The open, free-text comments were relatively straightforward to group into common themes. The first open item asked participants to make any other comments regarding these barriers or any others not mentioned in the survey. Thirty-four (37\%) participants responded. Most of these responses were additional comments about barriers already covered in the survey as follows: communication/teamwork $(n=7)$, staffing $(n=7)$, equipment $(n=6)$, staff attitude/culture $(n=5)$, patient attachments $(n$ 
$=1$ ), and medication $(n=1)$. A number of additional barriers to mobilization that were not in the survey were identified as follows: that physiotherapists were unavailable to help return patients to bed $(n=4)$, confused/violent/non-compliant/uncooperative patients $(n=2)$, air mattresses that make transfers difficult $(n=1)$, the need for staff to lead by example $(n=1)$, the need for more manual handling training $(n=1)$, and the need for staff education regarding the importance of mobilization $(n=1)$.

The final item of the survey asked participants to list two to three actions/interventions they thought would most effectively increase the frequency of mobilization of Royal Adelaide Hospital ICU patients. Seventy-four (80\%) participants responded. The themes most frequently identified were as follows: improve inter-disciplinary communication/teamwork $(n=39)$, increase staffing $(n=32)$, provide better/more equipment $(n=30)$, provide education to ICU staff regarding the benefits of mobilization $(n=22)$, improve documentation of mobility plans $(n=9)$, increase the assistance from physiotherapists with returning patients to bed $(n=$ $8)$, and identify/utilize mobility champions $(n=5)$.

\section{DISCUSSION}

As part of a broader quality improvement project, the aim of this study was to investigate the perceptions that ICU staff had regarding the barriers to mobilizing ICU patients. Overall, patient-related barriers (e.g. patient's medical condition and impending medical procedures/investigations) were perceived as having the most impact on the frequency of mobilization, followed closely by institutional-related barriers (e.g. time constraints and staff/equipment availability). The survey items that were perceived as least frequently impacting on the frequency of mobilization were that physiotherapists should have sole responsibility for all mobilization interventions for all ICU patients that compared to other aspects of care mobilization is unimportant, and that mobilization is not valued or promoted by ICU staff. Interventions that participants most frequently identified as having the potential to increase the frequency of mobilization in our ICU included improved inter-disciplinary communication/teamwork, increased staffing, better/more equipment, and provision of education regarding the benefits of mobilization.

Overall, our results were similar to previous ICU staff surveys where barriers to mobilization were considered.8,12,14 The survey by Appleton et al, which explored rehabilitation practices in Scottish ICUs, included items related to the factors most frequently limiting the provision of rehabilitation in ICU, with their sample comprising medical and physiotherapy staff. ${ }^{8}$ In keeping with our findings, the factors they most frequently reported as limiting the provision of rehabilitation were, in descending order, the severity of the patient's illness ( 72\%), insufficient funding $(\sim 68 \%)$, sedation $(\sim 63 \%)$, and insufficient equipment $(\sim 54 \%)$. King and Crowe undertook a postal survey investigating mobilization practices in Canadian ICUs, involving 81 physiotherapists, 18 nurses, and 15 physicians. ${ }^{12}$ As part of their survey, they investigated reasons ICU patients were not mobilized, with the main reasons being medical orders, unstable patient condition, unit policy, and too many lines/attachments. The most comprehensive study identified where ICU staff were surveyed regarding barriers to mobilization of ICU patients was by Koo. ${ }^{14}$ However, we were only able to identify an unpublished version of this study via the internet (unpublished thesis). ${ }^{14}$ In this study, Koo surveyed 194 physicians and 117 physiotherapists (representing a response rate of $68 \%$ ) working in Canadian ICUs. ${ }^{14}$ Similar to our findings, medical instability, excessive sedation, and risk of dislodgement of lines/devices were perceived as the most important patient-related barriers to the early mobilization of ICU patients, with obesity and cognitive impairment also important. The most frequently identified institutional-related barriers, similar to our findings, were a lack of written guidelines/protocols, insufficient equipment, and medical orders that were required pre-mobilization. ${ }^{14}$ In keeping with our results, Koo reported that the most frequently selected perceived provider barriers were limited physiotherapy/nursing staff. ${ }^{14}$ Koo also noted that more than half of respondents believed that early mobilization in the ICU was generally supported but not perceived as a priority of care; this is in contrast to our findings where mobilization was perceived as an important aspect of patient care. ${ }^{14}$ The findings of the current study, therefore, confirm and provide more detail regarding staff perceptions of the barriers to the mobilization of ICU patients.

\section{Limitations}

The most important limitations of the current study were the sample of convenience and low response rate. We deliberately restricted our sample to staff working in our ICU, rather than undertaking a national or international survey, as this survey formed part of a quality improvement project being undertaken in our ICU. Given that our findings are consistent with previous work, it is likely that similar barriers would be found in other ICUs, hence our results, while undertaken on a sample of convenience within a single ICU, are nevertheless of relevance to other ICUs. Whilst an excellent response rate was seen for physiotherapy staff, probably due to the small number of eligible participants $(n=4)$ and that this project was being driven by physiotherapists, the response rate from nursing staff was quite low $(27 \%)$; the reasons for this are unclear, but may include a lack of time/interest. We attempted to maximize participation by administering the survey in paper format (we had more confidence that the survey would reach the intended person when delivered via the internal mail system rather than relying on email notification and a web-based survey) and displaying reminder notices. Another limitation of the study was the imbalance between the professional groups who completed the survey; however, this imbalance is reflective of staffing ratios in our ICU. Furthermore, we believe it is a strength of our study that we attempted to recruit all ICU staff, irrespective of their profession, who were likely to be involved in

(C) The Internet Journal of Allied Health Sciences and Practice, 2015 
mobilization within our ICU. Whilst it would have been preferable to have used/modified a published survey, we were unable to identify one during the design stage. However, on completion of data collection, the unpublished work of Koo was identified during an internet search..$^{13,14}$ Whilst in retrospect, the survey designed by Koo ${ }^{14}$ could have been adapted for use in the current study, our purpose-designed survey covered similar content. We did not attempt to formally assess the validity and reliability of our purpose-designed survey. Whilst we acknowledge this as a limitation of our study, we believe our survey has both face and content validity.

\section{Implications for Clinical Practice}

Our findings, which concur with previous research, suggest that the main barriers to the implementation of mobilization in ICU are patient-related, followed closely by institutional-related barriers. Whilst the patient-related barriers to mobilization may be difficult to address, at least in the short term, some of the institutional-related barriers may be amenable to intervention. For example, increased resource allocation may overcome the barriers of time constraints and staff/equipment availability. Similarly, as highlighted by the participants in the open, free-text items, interventions aimed at improving inter-disciplinary communication/teamwork and provision of staff education regarding the benefits of mobilization may be effective at increasing the frequency of mobilization in ICU. Identifying barriers to mobilization that are amenable to intervention may enable the gap between evidence and practice to be narrowed, thus improving patients' functional outcomes and potentially decreasing ICU and hospital length of stay.

\section{Implications for Research}

Future research could be done to replicate this survey, or similar, in other ICUs both within Australia and internationally to see if the barriers to mobilization of ICU patients identified by ICU staff in this study are reproducible elsewhere. Research that attempts to measure the gap between evidence and its uptake into clinical practice with respect to the mobilization of ICU patients would also be of interest. As part of a larger quality improvement project, the results of this staff survey have been used to identify barriers to mobilization that are amenable to intervention in the short-term and strategies to overcome these barriers have been implemented in our ICU.

\section{CONCLUSION}

This study found that ICU staff perceived that patient-related factors were the most important barriers to the mobilization of ICU patients, followed closely by institutional-related barriers. Some of these barriers should be relatively easy to overcome, including improving inter-disciplinary communication, educating staff regarding the benefits of mobilization and improving documentation of mobility plans. The findings of this staff survey will be used to inform a quality improvement project which aims to increase the frequency of mobilization of ICU patients.

\section{REFERENCES}

1. Hodgson CL, Berney S, Harrold M, et al. Clinical review: early patient mobilization in the ICU. Crit Care. 2013;17(1):207. [PMID 23672747]

2. Hopkins RO, Spuhler VJ, Thomsen GE. Transforming ICU culture to facilitate early mobility. Crit Care Clin. 2007;23(1):81-96. [PMID 17307118]

3. $\quad$ Kress JP. Sedation and mobility: changing the paradigm. Crit Care Clin. 2013;29(1):67-75. [PMID 23182528]

4. Li Z1, Peng X, Zhu B, et al. Active mobilization for mechanically ventilated patients: a systematic review. Arch Phys Med Rehabil. 2013;94(3):551-61. [PMID 23127305]

5. Morris PE, Goad A, Thompson C, et al. Early intensive care unit mobility therapy in the treatment of acute respiratory failure. Crit Care Med. 2008;36(8):2238-43 [PMMID 18596631].

6. Needham DM, Korupolu R, Zanni JM, et al. Early physical medicine and rehabilitation for patients with acute respiratory failure: a quality improvement project. Arch Phys Med Rehabil. 2010;91(4):536-42. [PMID 20382284]

7. Stiller K. Physiotherapy in intensive care. An updated systematic review. Chest 2013;144(3):825-47. [PMID 23722822]

8. Appleton RTD, MacKinnon M, Booth MG, et al. Rehabilitation within Scottish intensive care units: a national survey. $J$ Intensive Care Soc. 2011;12(3):221-7.

9. Bahadur K, Jones G, Ntoumenopoulos G. An observational study of sitting out of bed in tracheostomised patients in the intensive care unit. Physiother. 2008:94:300-5.

10. Bassett RD, Vollman KM, Brandwene L, Murray T. Integrating a multidisciplinary mobility programme into intensive care practice (IMMPTP): a multicentre collaborative. Intensive Crit Care Nurs. 2012;28(2):88-97. [PMID 22227355]

11. Fan E. Critical illness neuromyopathy and the role of physical therapy and rehabilitation in critically ill patients. Respir Care. 2012;57(6):933-44. [PMID 22663968]

12. King J, Crowe J. Mobilization practices in Canadian critical care units. Physiother Can. 1998;50:206-11. 
13. Koo KKY. 2012. Facilitators and barriers to acute rehabilitation in the critically ill. Critical Care Canada Forum 2012. http://www.criticalcarecanada.com/presentations/2012/facilitators and barriers to acute rehabilitation in the critically ill.pdf (accessed May 2014).

14. Koo KKY. 2012. Survey of acute rehabilitation in Canadian intensive care units. 2012.

http://digitalcommons.mcmaster.ca/cgi/viewcontent.cgi?article=8559\&context=opendissertations (accessed May 2014).

15. Leditschke A, Green M, Irvine J, et al. What are the barriers to mobilizing intensive care patients? Cardiopulm Phys Ther J. 2012;23(1):26-9. [PMID 22807652]

16. Lipshutz AK, Gropper MA. Acquired neuromuscular weakness and early mobilization in the intensive care unit. Anesthesiology. 2013;118(1):202-15. [PMID 22929731]

17. Needham DM, Korupolu R. Rehabilitation quality improvement in an intensive care setting: implementation of a quality improvement model. Top Stroke Rehabil. 2010:17(4):271-81. [PMID 20826415]

18. Schweickert WD, Kress JP. Implementing early mobilization interventions in mechanically ventilated patients in the ICU. Chest. 2011;140(6):1612-7. [PMID 22147819]

19. Skinner EH, Berney S, Warrillow S, Denehy L. Rehabilitation and exercise prescription in Australian intensive care units. Physiother. 2008;94:220-9.

20. Vollman KM. Understanding critically ill patients hemodynamic response to mobilization: using the evidence to make it safe and feasible. Crit Care Nurs Q. 2013;36(1):17-27. [PMID 23221438]

21. Winkelman C, Peereboom K. Staff-perceived barriers and facilitators. Crit Care Nurse. 2010;30(2):S13-6. [PMID 20360441]

22. Zanni JM, Korupolu R, Fan E, et al. Rehabilitation therapy and outcomes in acute respiratory failure: an observational pilot project. J Crit Care. 2010:25(2):254-62. [PMID 19942399]

23. Hopkins RO, Suchyta MR, Farrer TJ, Needham D. Improving post-intensive care unit neuropsychiatric outcomes: understanding cognitive effects of physical activity. Am J Respir Crit Care Med. 2012;186(12):1220-8. [PMID 23065013]

24. Thomsen GE, Snow GL, Rodriguez L, Hopkins RO. Patients with respiratory failure increase ambulation after transfer to an intensive care unit where early activity is a priority. Crit Care Med. 2008;36(4):1119-24. [PMID 18379236] 
APPENDIX I

\section{SURVEY REGARDING THE BARRIERS TO MOBILISATION OF RAH ICU PATIENTS}

\section{Profession}

$\square$ Medical

$$
\begin{aligned}
& \square \text { Consultant } \\
& \square \text { Registrar }
\end{aligned}
$$

$\square$ Nursing

$$
\begin{aligned}
& \square \mathrm{CSC} \\
& \square \mathrm{ACSC} \\
& \square \mathrm{CN} \\
& \square \mathrm{CC} \mathrm{RN} \\
& \square \mathrm{RN} \\
& \square \mathrm{EN}
\end{aligned}
$$

$\square$ Physiotherapy

$$
\begin{aligned}
& \square \text { AHP3 } \\
& \square \text { AHP2 } \\
& \square \text { AHP1 }
\end{aligned}
$$

Is most (i.e. $>50 \%$ ) of your workload?

$\square$ Clinical

$\square$ Non-clinical

\section{Duration of working in the RAH ICU}

$$
\begin{aligned}
& \square<1 \text { year } \\
& \square 1-5 \text { years } \\
& \square>5 \text { years }
\end{aligned}
$$

Overall, please indicate on the visual analogue scale how well you think the RAH ICU is doing in terms of mobilising patients who are suitable for mobilisation.

Poorly, not many patients who should be mobilised are being mobilised
Excellently, all patients who should be mobilised are being mobilised

\section{BARRIERS TO EARLY PROGRESSIVE MOBILISATION}

The factors listed below are barriers to mobilisation that have been identified in previous studies. Please indicate on the visual analogue scale how frequently you believe these barriers prevent RAH ICU patients from being mobilised.

\section{Patient Barriers}

1. Medical orders (e.g. mobilisation contraindicated due to unstable spinal \#) 
2. Level of sedation

Never prevents mobilisation

Frequently prevents mobilisation

3. Reduced level of consciousness

\section{Never prevents}

mobilisation

Frequently prevents mobilisation

4. Agitation
Never prevents
Frequently prevents mobilisation mobilisation

5. Haemodynamic instability

Never prevents

mobilisation

Frequently prevents mobilisation

6. Respiratory instability (e.g. poor oxygenation, level of ventilatory support)

$\begin{array}{ll}\text { Never prevents } & \bullet \\ \text { mobilisation } & \text { Frequently prevents } \\ \text { mobilisation }\end{array}$

7. Weight / size

Never prevents mobilisation

Frequently prevents mobilisation

8. Potential for dislodgement of artificial airway (i.e. endotracheal tube or tracheostomy)

$\begin{array}{ll}\text { Never prevents } & \bullet \\ \text { mobilisation } & \text { Frequently prevents } \\ \text { mobilisation }\end{array}$

9. Potential for dislodgement of vascular lines, other attachments

$\begin{array}{ll}\text { Never prevents } & \bullet \\ \text { mobilisation } & \text { Frequently prevents } \\ \text { mobilisation }\end{array}$

10. Level of pain / discomfort / fatigue

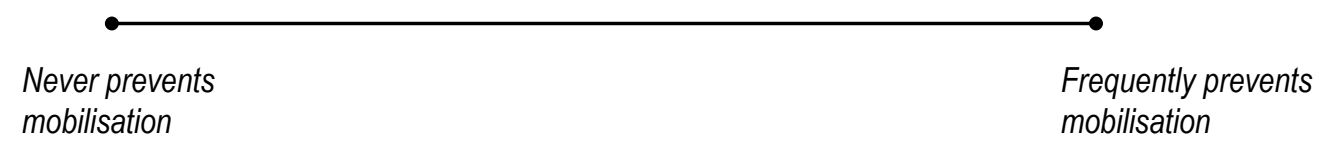

11. Impending medical procedure or investigation 
Never prevents

mobilisation
Frequently prevents mobilisation

12. Refused consent

Never prevents

Frequently prevents

mobilisation mobilisation

\section{Institutional Barriers}

1. Staff availability

$\begin{array}{ll}\begin{array}{l}\text { Never prevents } \\ \text { mobilisation }\end{array} & \begin{array}{l}\text { Frequently prevents } \\ \text { mobilisation }\end{array}\end{array}$

2. Staff expertise

Never prevents

mobilisation

Frequently prevents mobilisation

3. Staff willingness to help

$\begin{array}{ll}\text { Never prevents } & \bullet \\ \text { mobilisation } & \begin{array}{l}\text { Frequently prevents } \\ \text { mobilisation }\end{array}\end{array}$

4. Equipment availability
Never prevents
Frequently prevents mobilisation mobilisation

5. Equipment appropriateness

$\begin{array}{ll}\text { Never prevents } & \bullet \\ \text { mobilisation } & \begin{array}{l}\text { Frequently prevents } \\ \text { mobilisation }\end{array}\end{array}$

6. Risk of injury to staff

$\begin{array}{ll}\begin{array}{l}\text { Never prevents } \\ \text { mobilisation }\end{array} & \begin{array}{l}\text { Frequently prevents } \\ \text { mobilisation }\end{array}\end{array}$

7. Time constraints

Never prevents mobilisation
Frequently prevents mobilisation 
8. Imminent ward transfer

Never prevents mobilisation
Frequently prevents

mobilisation

\section{Other Barriers}

The statements listed below involve other barriers to mobilisation that have been identified in previous studies and from our own experience. Please indicate on the visual analogue scale how strongly you agree or disagree with statements in terms of preventing RAH ICU patients from being mobilised.

1. There are no clear recommendations in the RAH ICU regarding mobilisation

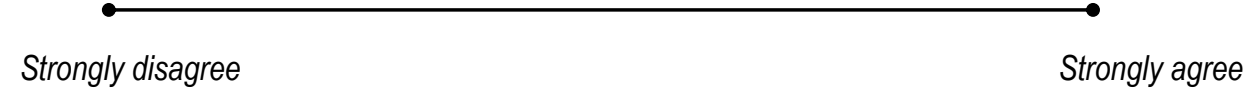

2. The RAH ICU culture does not promote and value mobilisation
Strongly disagree
Strongly agree

3. Compared to other aspects of patient care, mobilisation is unimportant

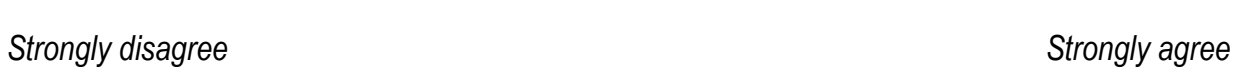

4. There is no clear definition of which profession should take responsibility for mobilising ICU patients

Strongly disagree

Strongly agree

5. There is poor inter-disciplinary communication regarding which patients need to mobilise, how they should mobilise and when this should occur

Strongly disagree $\quad$ Strongly agree

6. There is a lack of leadership championing the importance of mobilisation

Strongly disagree $\quad$ Strongly agree

7. The physiotherapists should have sole responsibility for all mobilisation interventions for all ICU patients

Strongly disagree $\longrightarrow$ 
Please make any other comments regarding these barriers to the mobilisation of RAH ICU patients or any others not mentioned.

What 2-3 things do you think could be done to most effectively increase the frequency of mobilisation of RAH ICU patients?

1.

2.

3.

Thank you for your participation 